\title{
INCISÃO SUBCOSTAL VERSUS LOMBOTOMIA PARA O TRASPLANTE RENAL: COMPARAÇÃO DAS DUAS ABORDAGENS CIRÚRGICAS.
}

\author{
Flank versus subcostal incision in kidney donors: a randomized clinical trial
} Wagner Eduardo Matheus'1, Adriano Fregonesi', Ricardo Reges Maia de Oliveira ${ }^{2}$, Victor Leitão², Gustavo Mendonça Borges²,
Ricardo Destro Saade', Cassio Riccetto', Marcelo Lopes de Lima ${ }^{1}$, Nelson Rodrigues Netto Júnior

\section{RESUMO}

Objetivos: Comparar tempo cirúrgico e de dissecção do hilo assim como o grau de dificuldade, condições do enxerto durante, após a dissecção e após o implante, complicações no pós-operatório, tempo de permanência hospitalar e quantificação da dor referida pelos pacientes submetidos à incisão subcostal versus lombotomia. Métodos: Realizado estudo prospectivo randomizado com 26 doadores renais, 15 foram submetidos a lombotomia (grupo 1) e 11 a incisão subcostal (grupo 2). Resultados: O tempo médio de cirurgia foi similar, 175 minutos e 176 minutos nos grupos $1 \mathrm{e} 2$, respectivamente, embora o tempo de dissecção do hilo e grau de dificuldade tenha sido maior no grupo 2 (44,3 versus 50 minutos). A perfusão dos enxertos no receptor foi melhor no grupo 1 . Não houve diferença significativa no tempo de permanência hospitalar (4,4 versus 4,2 dias) e com relação à dor esta foi maior no grupo 2. Conclusão: O resultados foram melhores no grupo de pacientes submetidos a lombotomia, porém verificou-se subjetivamente, que na incisão subcostal tem-se uma melhor abordagem do hilo, principalmente nos casos de anomalias.

Descritores: Doador Renal, Enxerto, Hilo, Subcostal.

Trabalho realizado na Universidade Estadual de Campinas (UNICAMP) - Faculdade de Ciências Médicas - Departamento de Cirurgia - Disciplina de Urologia.

1. Médico contratado da disciplina de Urologia da Unicamp;

2. Médico residente da disciplina de Urologia da Unicamp;

3. Prof. Titular da disciplina de Urologia da Unicamp.

Endereço para correspondência: Wagner Eduardo Matheus - MD. Universidade Estadual de Campinas (UNICAMP) - Faculdade de Ciências Médicas - Departamento de Cirurgia Disciplina de Urologia - Cidade Universitária Zeferino Vaz - Campinas - São Paulo - Brasil CEP 13081-970 - E-mail: wematheus@uol.com.br

Recebido em: 30/06/2004

Aceito: $08 / 12 / 2004$

\section{INTRODUÇÃO}

Tendo em vista a pouca disponibilidade de órgãos para transplante de doadores cadáveres, utiliza-se como alternativa a doação intervivos de rins. Deste modo, tenta-se melhorar a técnica cirúrgica com o objetivo de tornar o procedimento menos invasivo e seguro, visto que a nefrectomia no doador é um procedimento com morbidade de $10,7 \% .^{1}$ A exemplo desta afirmação, citamos o uso da videolaparoscopia para nefrectomia, porém, nem todos os serviços usam este recurso em sua rotina. Sendo assim, resolvemos comparar duas vias de acesso, lombotomia versus a incisão subcostal para o transplante renal intervivos, a fim de definir qual seria a melhor.

\section{Materiais e Métodos}

Este estudo prospectivo e randomizado analisou 26 doadores renais para transplante intervivos que foram divididos em dois grupos. O grupo 1 foi composto por 15 pacientes submetidos a lombotomia, sendo 7 homens e 8 mulheres com idade média de 37 anos (variando de 27 a 52 anos) e o Grupo 2 foi composto de 11 pacientes submetidos à incisão subcostal, sendo 06 homens e 05 mulheres com idade média de 33,8 anos (variando de 20 a 57 anos). Após os pacientes terem sido submetidos à anestesia geral com peridural contínua, foi feita uma lombotomia ou incisão subcostal para a nefrectomia do doador. 
Os grupos foram analisados e comparados com relação ao tempo de anestesia e de cirurgia, graus de dificuldade subjetivamente encontrados pela equipe, o tempo de dissecção do hilo renal, quantificação da perfusão durante a preparação renal para o implante em três subgrupos (total, $90-50 \%$ e $<50 \%$ ) e após o implante no receptor, classificando-a em imediata, 5-10 minutos e $>10$ minutos, assim como em total, $50-90 \%$ e $<50 \%$.

Todos os pacientes foram entrevistados um mês após a cirurgia e responderam a um questionário oral, onde graduavam a dor em três níveis: leve, moderada e intensa.

\section{RESULTADOS}

O tempo médio de anestesia foi maior no grupo 1 (217 versus 208 minutos); o tempo médio de cirurgia foi praticamente igual em ambos os grupos, 175 minutos e 176 minutos no grupo 1 e 2 , respectivamente; o tempo de dissecção do hilo foi menor no Grupo 1 (44,3 versus 50 minutos), o grau de dificuldade de dissecção do hilo também foi menor no Grupo 1; em 3 (20\%) pacientes a dissecção foi difícil, em 5 (33\%) houve moderada dificuldade e em 7 (46\%) foi fácil, ao passo que no Grupo 2, em 4 (36\%) pacientes a dissecção foi difícil, em 4 (36\%) houve moderada dificuldade e em 3 (27\%) foi fácil (tabela 1).

Tabela 1 - Comparação dos parâmetros cirúrgicos: lombotomia versus incisão subcostal para o doador no transplante renal $(n=26)$.

\begin{tabular}{lll}
\hline Parâmetros & Lombotomia $(\mathbf{n}=15)$ & Subcostal $(\mathbf{n = 1 1})$ \\
\hline Tempo de cirurgia $(\min )$ & $173(135-240)$ & $176(120-210)$ \\
$\begin{array}{l}\text { Tempo de dissecção } \\
\text { do hilo }\end{array}$ & $38,5(30-50)$ & $50(35-60)$ \\
Dificuldade na & & \\
dissecção do hilo & $20 \%$ difícil & $36 \%$ difícil \\
& 33\% moderada & $36 \%$ moderada \\
& $46 \%$ fácil & $27 \%$ fácil \\
\hline
\end{tabular}

A perfusão dos enxertos durante a preparação renal para o implante foi semelhante em ambos os grupos, porém a perfusão do enxerto no receptor foi melhor no Grupo 1 (tabelas 2 e 3): 73,3\% tiveram perfusão imediata e 26,6\% levaram de 5-10 minutos para perfundir; já no Grupo $2,27 \%$ tiveram perfusão imediata, $54 \%$ levaram de 5-10 minutos para perfundir e $19 \%$ levaram mais de 10 minutos para perfundir, sendo que um dos enxertos do Grupo 2 não perfundiu completamente, ficando uma área de isquemia de $10 \%$ do rim que era representada por uma artéria polar, que havia sido ligada na dissecção.

Tabela 2 - Comparação da Perfusão renal na preparação do enxerto para o implante: lombotomia versus incisão subcostal para o doador no transplante renal $(n=26)$.

\begin{tabular}{ccc}
\hline Perfusão & Lombotomia $(\mathbf{n = 1 5})$ & Subcostal $(\mathbf{n = 1 1})$ \\
\hline Total & $09(60 \%)$ & $08(73 \%)$ \\
$50-90 \%$ & $06(40 \%)$ & $03(27 \%)$ \\
$<50 \%$ & - & - \\
\hline
\end{tabular}

Tabela 3 - Comparação da perfusão renal após o implante do enxerto: lombotomia versus incisão subcostal para o doador no transplante renal $(n=26)$.

\begin{tabular}{ccc}
\hline Perfusão & Lombotomia $(\mathbf{n}=\mathbf{1 5})$ & Subcostal $(\mathbf{n}=\mathbf{1 1})$ \\
\hline Imediata & $11(73,3 \%)$ & $03(27 \%)$ \\
$5-10$ min. & $04(26,6 \%)$ & $06(54 \%)$ \\
$>10$ min. & - & $02(19 \%)$ \\
Não houve & - & - \\
\hline
\end{tabular}

No transoperatório, a única complicação ocorreu no grupo dos pacientes submetidos à incisão subcostal: uma lesão de veia cava durante a ligadura de duas veias renais, porém o sangramento foi controlado facilmente e não houve necessidade de transfusão de hemoderivados. Nenhum paciente necessitou de transfusão sanguínea.

No pós-operatório, os pacientes do Grupo 1 tiveram menos dor, pois a necessidade de aplicações de morfina peridural foi 3,0 versus 3,8 aplicações e na avaliação, a dor foi forte em $13 \%$, moderada em $66,6 \%$ e leve em $20 \%$ no Grupo 1 e $36 \%$ tiveram dor forte e $64 \%$ moderada no Grupo 2.

A morbidade no pós-operatório, no grupo de pacientes submetidos a lombotomia, foi de $6,6 \%$, representada por um caso de infecção de ferida operatória; não houve morbidade no grupo de pacientes submetidos à incisão subcostal. A morbidade encontrada em nossa amostragem está dentro do que é relatado na literatura, que é de aproximadamente 3,5 \%.2 Não houve êxito letal em quaisquer dos grupos.

O tempo médio de internação foi semelhante em ambos grupos $(5,2$ versus 5,0 dias), não houve complicações nem transfusões de sangue ou hemoderivados no intra-operatório ou no pós-operatório.

Não houve perda de enxerto por problemas cirúrgicos ou clínicos em ambos os grupos durante o seguimento, de modo que todos os rins continuam funcionando adequadamente até a presente data.

\section{DISCUSSÃO}

A doação de rim intervivos ainda é bastante utilizada em nosso meio, devido à limitação de doadores cadáveres. Deste modo, há uma busca contínua de melhoria da técnica cirúrgica, o que nos fez comparar a incisão subcostal e a lombotomia como via de acesso para nefrectomia na doação intervivos.

Como podemos observar, o acesso através da lombotomia obteve melhores resultados em relação ao tempo e grau de dificuldade de dissecção do hilo renal e na avaliação da dor no pós-operatório, como também uma melhor perfusão renal, porém, sob nosso ponto de vista, o acesso subcostal também tem suas vantagens, como no caso de múltiplas artérias renais, onde este acesso fornece uma ampla visualização do hilo e menor manipulação renal, causando menor espasmo e também nos casos de pacientes com doença pulmonar obstrutiva, pois não há ressecção da costela, proporcionando, portanto, uma melhor ventilação. Deste modo não vemos o acesso subcostal como uma opção a ser descartada e sim acrescentada ao arsenal de recursos a serem utilizados no transplante renal com indicações precisas.

\section{CONCLUSÃO}

O tempo de dissecção, a perfusão renal e a avaliação subjetiva da dor foram melhores no grupo de pacientes submetidos à lombotomia, embora tenha sido verificado que na incisão subcostal direita, a abordagem do hilo, principalmente nos casos de anomalias como rim com múltiplas artérias, ${ }^{2,3,4}$ seja melhor do que a lombotomia. 


\section{ABSTRACT}

Objective: To compare flank versus subcostal incision in kidney donors considering surgery and hilum dissection duration, as well as difficulty and conditions of the graft during harvesting and after transplantation. Post-operative complications, hospital stay and postoperative pain were also compared. Methods: Twenty-six consecutive kidney donors were evaluated in a prospective and randomized manner, comparing flank and anterior subcostal incision, being 15 in the first (group 1 - flank incision) and 11 in the latter (group 2 - anterior subcostal incision). Results: Average surgery length was similar, 175 versus 176 minutes respectively, although difficulty and dissection time of the hilum were greater in group 02 (44.3 versus 50 minutes). Reperfusion was better in the group 1 than in the group 2. The length of hospitalization was similar in both groups (4.4 and 4.2 days respectively). The group 2 had more postoperative pain than group 1. Conclusions: Although flank incision had better results, the subcostal incision still have to be considered, mainly in cases of vascular anomalies and chronic obstructive pulmonary disease.

Key words: kidney donor, graft, hilum, subcostal.

\section{REFERÊNCIAS}

Baier PK, Pisarski P, Wimmenauer S, Kirste G. Kidney donation by living donors Surgical procedure. Zentralbl Chir 1999; 124(8): 729-33

2. Ruiz R, Novick AC, Braun WE, Montague DK, Stewart BH. Transperitoneal live donor nephrectomy. J Urol 1980 Jun;123(6):819-21

3. Connor WT, Van Buren CT, Floyd M, Kahan BD. Anterior extraperitoneal donor nephrectomy. J Urol 1981 Oct; 126(4): 443-7

4. Cohen EL, Kim SW, Schanzer H, Burrows L. Living-related donor nephrectomy by eleventh rib intraperitoneal extrapleuralincision. Urology 1985 Jun; 25(6): $579-81$

5. Adamec M. Removal of the kidney from a related transplant donor using subcostal lumbotomy. Rozhl Chir 1995 Sep; 74(6): 302-4 